\title{
Quantum non-demolition detection of single microwave photons in a circuit
}

\author{
B. R. Johnson', M. D. Reed', A. A. Houck ${ }^{2}$, D. I. Schuster', Lev S. Bishop', E. Ginossar', \\ J. M. Gambetta ${ }^{3}$, L. DiCarlo', L. Frunzio ${ }^{1}$, S. M. Girvin ${ }^{1}$ and R. J. Schoelkopf ${ }^{1 \star}$
}

Thorough control of quantum measurement is key to the development of quantum information technologies. Many measurements are destructive, removing more information from the system than they obtain. Quantum non-demolition (QND) measurements allow repeated measurements that give the same eigenvalue'. They could be used for several quantum information processing tasks such as error correction ${ }^{2}$, preparation by measurement ${ }^{3}$ and one-way quantum computing ${ }^{4}$. Achieving QND measurements of photons is especially challenging because the detector must be completely transparent to the photons while still acquiring information about them ${ }^{5,6}$. Recent progress in manipulating microwave photons in superconducting circuits ${ }^{7-9}$ has increased demand for a QND detector that operates in the gigahertz frequency range. Here we demonstrate a QND detection scheme that measures the number of photons inside a high-quality-factor microwave cavity on a chip. This scheme maps a photon number, $n$, onto a qubit state in a single-shot by means of qubit-photon logic gates. We verify the operation of the device for $n=0$ and 1 by analysing the average correlations of repeated measurements, and show that it is $90 \%$ QND. It differs from previously reported detectors ${ }^{5,8-11}$ because its sensitivity is strongly selective to chosen photon number states. This scheme could be used to monitor the state of a photon-based memory in a quantum computer.

Several teams have engineered detectors that are sensitive to single microwave photons by strongly coupling atoms (or qubits) to high-quality-factor (high-Q) cavities. This architecture, known as cavity quantum electrodynamics (cavity QED), can be used in various ways to detect photons. One destructive method measures quantum Rabi oscillations of an atom or qubit resonantly coupled to the cavity ${ }^{8-10}$. The oscillation frequency is proportional to $\sqrt{n}$, where $n$ is the number of photons in the cavity, so this method essentially measures the time-domain swap frequency.

Another method uses a dispersive interaction to map the photon number in the cavity onto the phase difference of a superposition of atomic states $\left(|g\rangle+\mathrm{e}^{i \phi}|e\rangle\right) / \sqrt{2}$. Each photon number $n$ corresponds to a different phase $\phi$, so repeated Ramsey experiments ${ }^{5}$ can be used to estimate the phase and extract $n$. This method is QND, because it does not exchange energy between the atom and photon. However, as the phase cannot be measured in a single operation, it does not extract full information about a particular Fock state $|n\rangle$ in a single interrogation. Nonetheless, using Rydberg atoms in cavity QED, remarkable experiments have shown quantum jumps of light and the collapse of the photon number by measurement ${ }^{5,12}$.

Here we report a new method that implements a set of programmable controlled-NOT (CNOT) operations between an $n$-photon Fock state and a qubit, asking the question 'are there exactly $n$ photons in the cavity?' A single interrogation consists of applying one such CNOT operation and reading-out the resulting qubit state. To do this we use a quasi-dispersive qubit-photon interaction that causes the qubit transition frequency to depend strongly on the number of photons in the cavity. Consequently, frequency control of a pulse implements a conditional $\pi$ rotation on the qubit - the qubit state is inverted if and only if there are $n$ photons in the storage cavity. To ensure that this is QND, the qubit and storage cavity are adiabatically decoupled before carrying out a measurement of the qubit state.

To realize this method we extend circuit-based cavity QED (ref. 13) by coupling a single transmon qubit ${ }^{14,15}$ simultaneously to two cavities. In this scheme, the transmon qubit is used to interrogate the state of one cavity; then the transmon is measured with the other cavity. This separation of functions allows one cavity to be optimized for coherent storage of photons (high-Q) and the other for fast qubit readout (low-Q). Related work by Leek et al. ${ }^{16}$ realized a single transmon coupled to two modes of a single cavity, where the two modes were engineered to have very different quality factors. A schematic of the two-cavity device is shown in Fig. 1a. The cavities are realized as $\mathrm{Nb}$ coplanar waveguide resonators with $\lambda / 2$ resonances at $\omega_{s} / 2 \pi=5.07 \mathrm{GHz}$ and $\omega_{\mathrm{m}} / 2 \pi=6.65 \mathrm{GHz}$, respectively. The cavities are engineered, by design of the capacitors $C_{\mathrm{s}}$ and $C_{\mathrm{m}}$, to have very different decay rates $\left(\kappa_{\mathrm{s}} / 2 \pi=50 \mathrm{kHz}\right.$ and $\left.\kappa_{\mathrm{m}} / 2 \pi=20 \mathrm{MHz}\right)$ so that the qubit state can be measured several times per photon lifetime in the storage cavity. By having these cavities at different frequencies, the fast decay of the readout cavity does not adversely affect photons in the storage cavity. A transmon qubit is end-coupled to the two cavities, with finger capacitors controlling the individual coupling strengths $\left(g_{\mathrm{s}} / 2 \pi=70 \mathrm{MHz}\right.$ and $\left.g_{\mathrm{m}} / 2 \pi=83 \mathrm{MHz}\right)$. The usual shunt capacitor between the transmon islands is replaced with capacitors to the ground planes to reduce direct coupling between the cavities. Furthermore, a flux bias line ${ }^{17}$ allows fast, local control of the magnetic field near the transmon. This facilitates manipulations of the detunings $\Delta_{\mathrm{s}}=\omega_{g, e}-\omega_{\mathrm{s}}$ and $\Delta_{\mathrm{m}}=\omega_{g, e}-\omega_{\mathrm{m}}$ between the transmon and cavities, where we use the convention of labelling the transmon states from lowest to highest energy as $(g, e, f, h, \ldots)$.

To achieve high photon-number selectivity of the CNOT operations, there must be a large separation between the numberdependent qubit transition frequencies. To obtain this, we use small detunings $\left(\Delta_{s} / g_{s}<10\right)$ between the qubit and storage cavity. Figure 2 shows spectroscopy in this quasi-dispersive regime as a function of flux bias when the storage cavity is populated with a coherent state $(\langle\hat{n}\rangle \sim 1)$. A numerical energy-level calculation

${ }^{1}$ Departments of Physics and Applied Physics, Yale University, New Haven, Connecticut 06511, USA, ${ }^{2}$ Department of Electrical Engineering, Princeton University, Princeton, New Jersey 08544, USA, ${ }^{3}$ Institute for Quantum Computing and Department of Physics and Astronomy, University of Waterloo, Waterloo, Ontario N2L 3G1, Canada. *e-mail: robert.schoelkopf@yale.edu. 

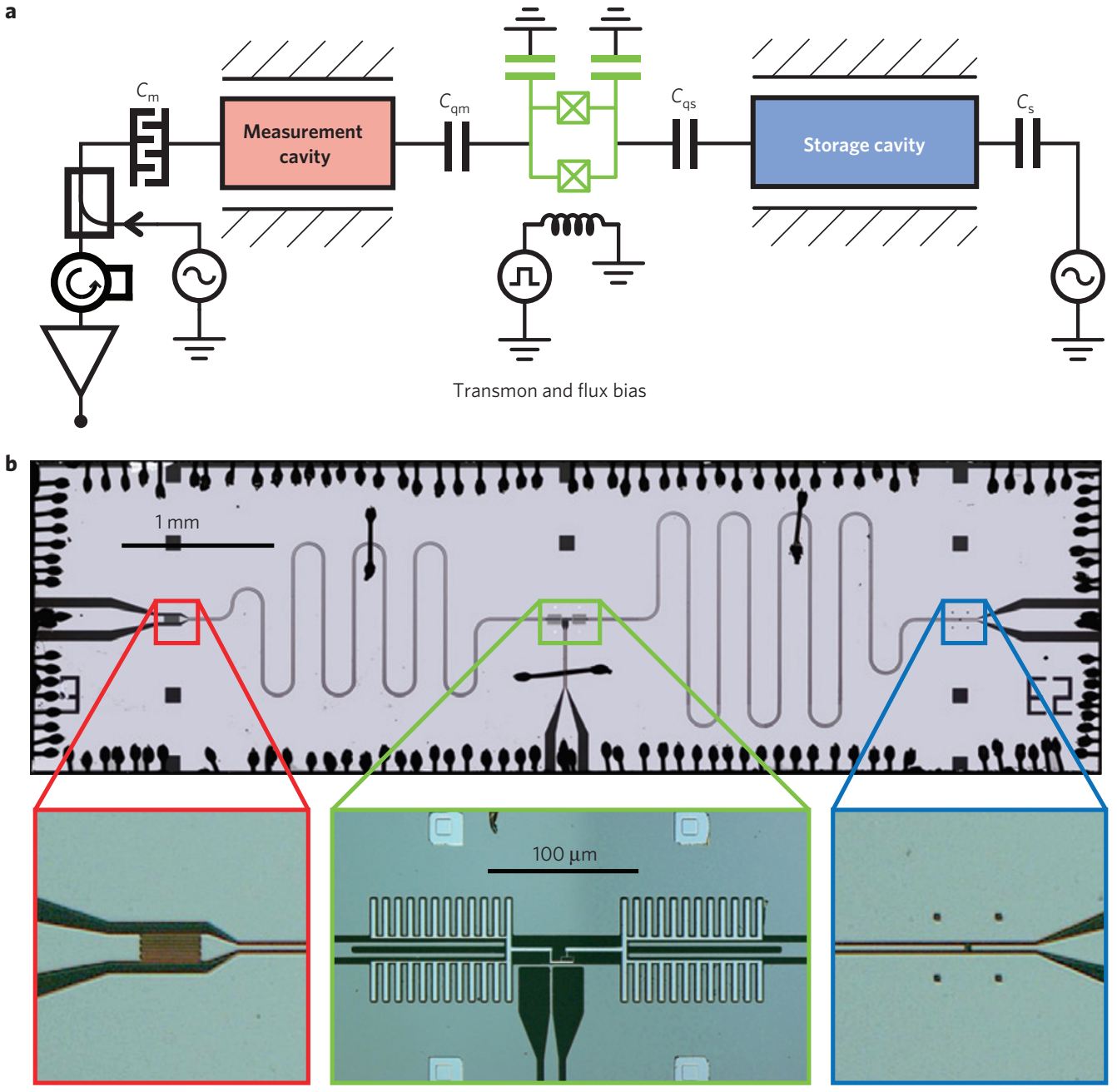

Figure 1 | Circuit schematic and device. a, Circuit schematic showing two cavities coupled to a single transmon qubit. The measurement cavity is probed in reflection by sending microwave signals through the weakly coupled port of a directional coupler. A flux bias line allows for tuning of the qubit frequency on nanosecond timescales. $\mathbf{b}$, Implementation on a chip, with a $\omega_{\mathrm{m}} / 2 \pi=6.65 \mathrm{GHz}$ measurement cavity on the left and its large coupling capacitor (red), and a $\omega_{\mathrm{s}} / 2 \pi=5.07 \mathrm{GHz}$ storage cavity on the right with a much smaller coupling capacitor (blue). A transmon qubit (green) is strongly coupled to each cavity, with $g_{\mathrm{s}} / 2 \pi=70 \mathrm{MHz}$ and $g_{\mathrm{m}} / 2 \pi=83 \mathrm{MHz}$. It has a charging energy $E_{\mathrm{C}} / 2 \pi=290 \mathrm{MHz}$ and maximal Josephson energy $E_{\mathrm{J}} / 2 \pi \approx 23 \mathrm{GHz}$. At large detunings from both cavities, the qubit coherence times are $T_{1} \approx T_{2} \approx 0.7 \mu \mathrm{s}$.

is overlaid, showing the positions of various transitions. We define $\omega_{g, e}^{n}$ as the photon-number-dependent transition frequency $|n, g\rangle \rightarrow|n, e\rangle$. Other transitions, such as $|2, g\rangle \rightarrow|0, h\rangle$, are allowed because of the small detuning. Fortunately, we also see that the separation between $\omega_{g, e}^{0}$ and $\omega_{g, e}^{1}$ grows rapidly to order $\sim 2 g_{\mathrm{s}}=140 \mathrm{MHz}$ as the qubit approaches the storage cavity.

To test the photon meter, we generate single photons in the storage cavity with an adiabatic protocol. Our method uses the avoided crossing between the $|0, e\rangle$ and $|1, g\rangle$ levels to convert a qubit excitation into a photon. The preparation of a photon begins with the qubit detuned below the storage cavity $\left(\Delta_{\mathrm{s}} \simeq-3 g_{\mathrm{s}}\right)$, where we apply a $\pi$-pulse to create the state $|0, e\rangle$. We then adiabatically tune the qubit frequency through the avoided crossing with the storage cavity, leaving the system in the state $|1, g\rangle$. The sweep rate is limited by Landau-Zener transitions that keep the system in $|0, e\rangle$. The preparation protocol changes the qubit frequency by $600 \mathrm{MHz}$ in $50 \mathrm{~ns}$, giving a spurious transition probability less than $0.1 \%$ (calculated with a multi-level numerical simulation). This protocol actually allows for the creation of arbitrary superpositions of $|0, g\rangle$ and $|1, g\rangle$ by changing the rotation angle of the initial pulse. For example, if we use a $\pi / 2$-pulse, after the sweep the system ends up in the state $\left(|0, g\rangle+\mathrm{e}^{i \phi}|1, g\rangle\right) / \sqrt{2}$, where $\phi$ is determined by the rotation axis of the $\pi / 2$-pulse. One could also use a resonant swap scheme, which has been successfully used to create Fock states ${ }^{9}$ up to $|n=15\rangle$. The method used here has the advantage of being very robust to timing errors.

After the photon is prepared in the storage cavity, the qubit frequency is adjusted such that $\Delta_{\mathrm{s}} / g_{\mathrm{s}} \simeq 5$. At this detuning, the separation between $\omega_{g, e}^{0}$ and $\omega_{g, e}^{1}$ is $\sim 65 \mathrm{MHz}$. In Fig. 3a, we show pulsed spectroscopy at this detuning for several rotation angles of the initial preparation pulse. We observe well-resolved dips in the reflected phase of a pulsed signal sent at the measurement cavity frequency. The locations of these dips correspond to the qubit transition frequencies for $n=0\left(\omega_{g, e}^{0}\right)$ and $n=1\left(\omega_{g, e}^{1}\right)$, and the relative heights match expectations from the different preparation-pulse rotations (for example, a $\pi / 2$-pulse results in equal-height signals).

To show selective driving of these transitions, we carry out Rabi experiments at $\omega_{g, e}^{0}$ and $\omega_{g, e}^{1}$ for the cases where we prepare $|0, g\rangle$ and $|1, g\rangle$. In each experiment we ensemble average measurements of the resulting qubit state after further decoupling the qubit from the storage cavity. For the $|0, g\rangle$ case (Fig. $3 b$ ) there is a large-amplitude oscillation when the drive is at $\omega_{g, e}^{0}\left(\right.$ red, $\left.R_{0}(\theta)\right)$ and almost no oscillation when the drive is at $\omega_{g, e}^{1}$ (blue, $R_{1}(\theta)$ ). When we prepare $|1, g\rangle$ the situation is reversed (Fig. 3c); however, 


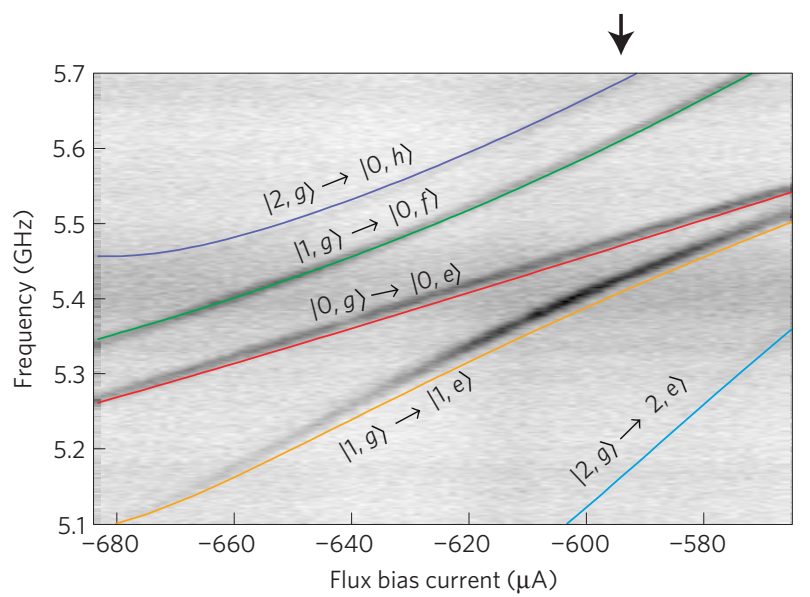

Figure 2 | Pulsed spectroscopy with a coherent state in storage cavity $(\langle n\rangle \approx 1)$ versus qubit-cavity detuning $\Delta_{\mathrm{s}}=\omega_{g, \mathrm{e}}-\omega_{\mathrm{s}}$. Calculated transition frequencies are overlaid in colour. The red and orange lines are the $|g\rangle \leftrightarrow|e\rangle$ transitions of the qubit when $n=0$ and 1, respectively. Transitions to higher transmon levels $(|f\rangle$ and $|h\rangle)$ are visible because of the small detuning. The arrow indicates the flux bias current used during the CNOT operations. in this case the residual oscillation of $R_{0}(\theta)$ (red) is substantial because of small errors in the preparation of $|1, g\rangle$ associated with the initial rotation of the qubit and, more importantly, the $\sim 10 \%$ probability of energy decay during the subsequent adiabatic sweep through the cavity.

The responses $R_{i}(\theta)$ are a result of driving $\omega_{g, e}^{i}$ and the far off-resonant drive of $\omega_{g, e}^{j}$, where $j \neq i$. The cross-talk is seen in the small residual oscillation of $R_{1}(\theta)$ in Fig. 3b. In the Supplementary Information, we derive a method for extracting a selectivity and preparation fidelity from these data, giving a selectivity $\geq 95 \%$ for both interrogations and a preparation fidelity of $|\langle n=1 \mid \psi\rangle|^{2} \approx 88 \%$. These numbers were confirmed by doing equivalent experiments over a range of preparation-pulse rotation angles between 0 and $2 \pi$ (not shown).

If $\pi$-pulses are used in the interrogation step, measurement results of the average qubit state directly correlate with the probability of being in the states $|n=0\rangle$ or $|n=1\rangle$. Details of the scaling needed to do this transformation when the selectivity is $<100 \%$ are presented in the Supplementary Information. These are the desired CNOT operations of the photon meter. If we now insert a variable delay before interrogating, we find that $P_{0}\left(P_{1}\right)$, the probability of being in $|n=0\rangle(|n=1\rangle)$, decays exponentially towards $1(0)$, as shown by the red (orange) trace in Fig. $4 \mathrm{~b}$. The decay constant of $T_{1} \simeq 3.11 \pm 0.02 \mu$ s agrees with the linewidth of

a



b

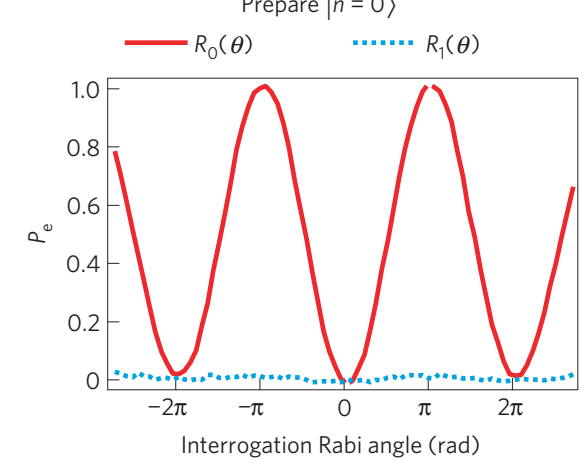

c

Prepare $|n=1\rangle$

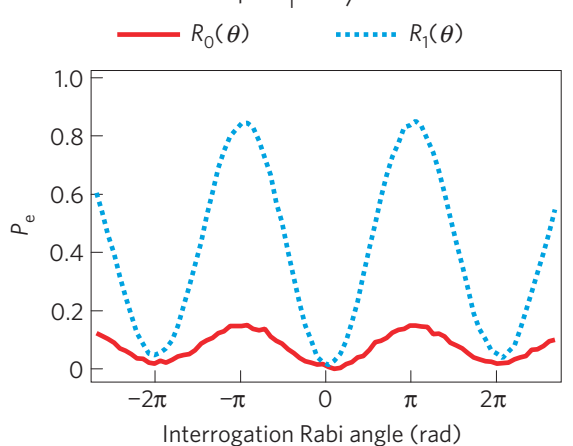

Figure 3 | Single photon preparation and CNOT selectivity. a, Pulsed spectroscopy versus Rabi angle of the preparation pulse, showing the reflected phase of a pulse at the measurement cavity frequency after a $\sim 80 \mathrm{~ns}$ pulse near the qubit frequency. Traces are offset vertically for clarity and labelled with the rotation angle of the control pulse used in the preparation step. The dips correspond to $\omega_{\mathrm{g}, \mathrm{e}}^{0} \approx 5.47 \mathrm{GHz}$ and $\omega_{\mathrm{g}, \mathrm{e}}^{1} \approx 5.41 \mathrm{GHz}$, respectively. b,c, Rabi driving the qubit transitions after preparing $|n=0\rangle(\mathbf{b})$ and $|n=1\rangle(\mathbf{c})$. The red (blue) traces show the measured qubit excited state probability after applying an interrogation Rabi pulse with varying angle at $\omega_{g, e}^{0}\left(\omega_{g, e}^{1}\right)$. The residual oscillation of $R_{1}(\theta)$ in $\mathbf{c}$ is mostly due to preparation infidelity. 

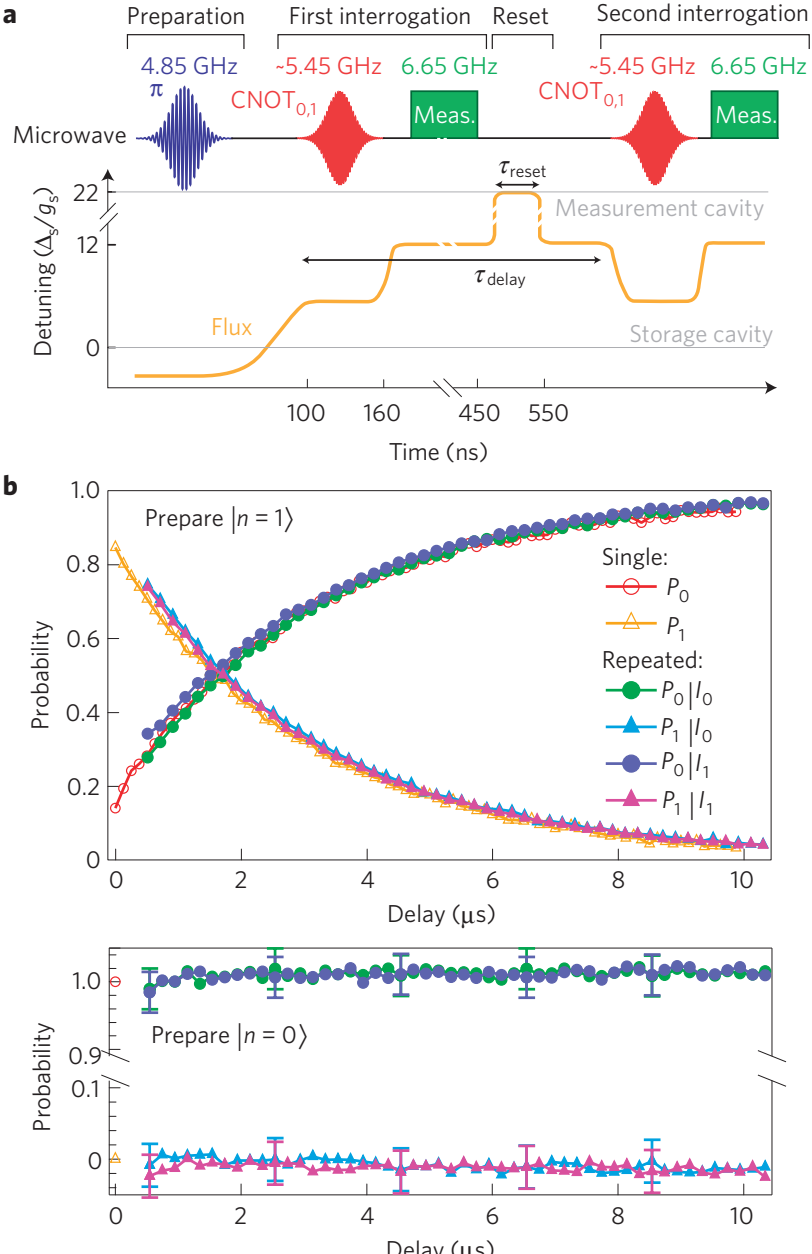

c

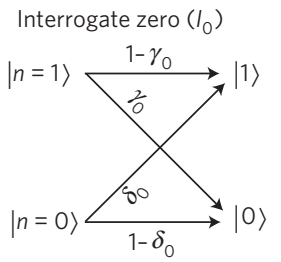

Interrogate one $\left(I_{1}\right)$



Figure 4 | Repeated measurements of photons. a, Experiment protocol. A microwave pulse and adiabatic sweep load a single photon into the storage cavity in the preparation step. This photon is interrogated repeatedly by number-selective CNOT gates on the qubit, followed by adiabatic decoupling, qubit readout and reset. $\mathbf{b}$, Single and repeated interrogation after preparing $|n=1\rangle$ (top) or $|n=0\rangle$ (bottom), ensemble averaged over $\sim 50,000$ iterations. The near-perfect overlap between single and repeated results demonstrates that the protocol is highly QND. The estimated uncertainty is the same for each point, so only a few representative error bars are shown for clarity (see Supplementary Information for error estimates).c. Transition probability diagrams for the interrogate $n=0\left(I_{0}\right)$ and interrogate $n=1\left(l_{1}\right)$ processes. We extract $\gamma_{0}\left(\gamma_{1}\right)=1(10) \pm 3 \%$ and $\delta_{0}\left(\delta_{1}\right)=7(3) \pm 3 \%$.

the storage cavity, $1 / \kappa_{\mathrm{s}}=1 /(2 \pi 50 \mathrm{kHz})=3.2 \pm 0.1 \mu \mathrm{s}$, measured in a separate, low-power $(\bar{n} \sim 1)$ reflection experiment.

Strong QND measurements are projective, such that if the measurement observable commutes with the Hamiltonian, the system will remain in an eigenstate of both operators between measurements. Consequently, comparing the results of successive interrogations provides a mechanism to test whether a particular protocol causes perturbations on the system beyond the expected projection. Here, we compare only ensemble average results, because the single-shot qubit readout fidelity for the device is $\sim 55 \%$. This is sufficient to reveal processes that change the photon number, and technical improvements to interrogation speed or qubit readout fidelity should allow for real-time monitoring of the photon state.

The protocol cannot be repeated immediately, though, because the first interrogation may leave the qubit in the excited state. To circumvent this problem, we use the fast decay rate of the measurement cavity to cause the qubit to spontaneously decay into the $50 \Omega$ environment. The 'reset' protocol brings the qubit into resonance with the measurement cavity for a time, $\tau_{\text {reset }}=50 \mathrm{~ns}$, which is sufficient to reset the qubit with probability $\sim 98 \%$. The procedure is described in detail in ref. 18.

After resetting the qubit, we can interrogate a second time. The full protocol for a repeated interrogation sequence is shown in Fig. 4a. The combination of a $\mathrm{CNOT}_{0}\left(\mathrm{CNOT}_{1}\right)$, a qubit measurement and a qubit reset define an interrogation process $I_{0}\left(I_{1}\right)$. Data for the four possible combinations of interrogating $|n=0\rangle$ and $|n=1\rangle$ are shown in Fig. $4 \mathrm{~b}$ as a function of delay between the first and second interrogations. The data are ensemble averaged over all results from the first interrogation, so we do not observe projection onto number states. Instead, we again observe exponential decay, where the result of the second measurement is essentially indistinguishable from the first, indicating that the interrogation is highly QND.

Small deviations between first and second interrogations stem from finite photon lifetime in the storage cavity and non-QND processes that cause transitions to other photon numbers (Fig. 4c). A single interrogation process takes $\sim 550 \mathrm{~ns}$, which accounts for the time gap between the single and repeated interrogation points in Fig. 4b. Recording the second interrogation results for different delays allows us to subtract the effect of photon $T_{1}$ and calculate the transition probabilities for the $I_{0}$ and $I_{1}$ processes $^{19}$. In principle, $I_{0}$ and $I_{1}$ can cause transitions to photon numbers outside the $n \in\{0,1\}$ manifold; however, the absence of statistically significant deviations from $P_{0}+P_{1}=1$ suggests that any such effects are negligible. Instead, we consider only transitions from $|n=1\rangle \rightarrow|n=0\rangle$ or $|n=0\rangle \rightarrow|n=1\rangle$, characterized by the probabilities $\gamma_{i}$ and $\delta_{i}$, respectively, where $i$ references the interrogation process $I_{0}$ or $I_{1}$. We observe $\gamma_{0}\left(\gamma_{1}\right)=1(10) \pm 3 \%$ and $\delta_{0}\left(\delta_{1}\right)=7(3) \pm 3 \%$, demonstrating that this protocol is highly QND.

The protocol presented here is a fast and highly QND measurement of single photons, which we believe can be extended to detect higher photon numbers. It should be possible to demonstrate the projective nature of the interrogation and create highly non-classical states of light by post-selection, and eventually with higher fidelity readout it should be possible to observe quantum jumps of light in a circuit.

Received 12 March 2010; accepted 25 May 2010; published online 20 June 2010

\section{References}

1. Braginsky, V. B. \& Khalili, F. Y. Quantum nondemolition measurements: The route from toys to tools. Rev. Mod. Phys. 68, 1-11 (1996).

2. Steane, A. M. Simple quantum error-correcting codes. Phys. Rev. A 54, 4741-4751 (1996).

3. Ruskov, R. \& Korotkov, A. N. Entanglement of solid-state qubits by measurement. Phys. Rev. B 67, 241305 (2003).

4. Raussendorf, R. \& Briegel, H. J. A one-way quantum computer. Phys. Rev. Lett. 86, 5188-5191 (2001).

5. Guerlin, C. et al. Progressive field-state collapse and quantum non-demolition photon counting. Nature 448, 889-893 (2007).

6. Gambetta, J. et al. Qubit-photon interactions in a cavity: Measurement induced dephasing and number splitting. Phys. Rev. A 74, 042318 (2006).

7. Houck, A. A. et al. Generating single microwave photons in a circuit. Nature 449, 328-331 (2007).

8. Hofheinz, M. et al. Generation of Fock states in a superconducting quantum circuit. Nature 454, 310-314 (2008). 
9. Hofheinz, M. et al. Synthesizing arbitrary quantum states in a superconducting resonator. Nature 459, 546-549 (2009).

10. Brune, M. et al. Quantum Rabi oscillation: A direct test of field quantization in a cavity. Phys. Rev. Lett. 76, 1800-1803 (1996).

11. Schuster, D. I. et al. Resolving photon number states in a superconducting circuit. Nature 445, 515-518 (2007).

12. Gleyzes, S. et al. Quantum jumps of light recording the birth and death of a photon in a cavity. Nature 446, 297-300 (2007).

13. Schoelkopf, R. J. \& Girvin, S. M. Wiring up quantum systems. Nature 451, 664-669 (2008).

14. Koch, J. et al. Charge-insensitive qubit design derived from the Cooper pair box. Phys. Rev. A 76, 042319 (2007).

15. Schreier, J. A. et al. Suppressing charge noise decoherence in superconducting charge qubits. Phys. Rev. B 77, 180502 (2008).

16. Leek, P. J. et al. Cavity quantum electrodynamics with separate photon storage and qubit readout modes. Phys. Rev. Lett. 104, 100504 (2010).

17. DiCarlo, L. et al. Demonstration of two-qubit algorithms with a superconducting quantum processor. Nature 460, 240-249 (2009).

18. Reed, M. D. et al. Fast reset and suppressing spontaneous emission of a superconducting qubit. Appl. Phys. Lett. 96, 203110 (2010).
19. Lupasçu, A. et al. Quantum non-demolition measurement of a superconducting two-level system. Nature Phys. 3, 119-123 (2007).

\section{Acknowledgements}

We thank J. Chow and M. Devoret for helpful discussions. This work was supported by NSF grants DMR-0603369 and PHY-0653073. J.M.G. was supported by a CIFAR Junior Fellowship, MITACS, MRI and NSERC. L.F. was partially supported by CNR-Istituto di Cibernetica.

\section{Author contributions}

B.R.J. and M.D.R. carried out measurements and data analysis. A.A.H., D.I.S. and L.D. provided further experimental contributions. L.S.B., E.G. and J.M.G. provided theoretical contributions. Devices were fabricated by B.R.J., L.F. and L.D. The experiment was designed by B.R.J., A.A.H., D.I.S., J.M.G., S.M.G. and R.J.S.

\section{Additional information}

The authors declare no competing financial interests. Supplementary information accompanies this paper on www.nature.com/naturephysics. Reprints and permissions information is available online at http://npg.nature.com/reprintsandpermissions. Correspondence and requests for materials should be addressed to R.J.S. 